\title{
Physics of THz Field-Effect Transistors
}

\author{
J. ŁUSAKOWSKI \\ Institute of Experimental Physics, University of Warsaw, Hoża 69, 00-681 Warsaw, Poland \\ Field-effect transistors are nowadays considered as possible elements of $\mathrm{THz}$ detection and emission systems. \\ Their $\mathrm{THz}$ performance is governed by excitations of two-dimensional plasma in the transistor channel. The \\ paper discusses peculiarities of the photon-plasmon coupling mechanism in field-effect transistors and puts it in \\ the perspective of classical investigation of plasma excitations in two-dimensional systems.
}

PACS: 73.20.Mf, 52.75.-d

\section{Introduction}

Emerging applications are the driving force for development of $\mathrm{THz}$ detectors and emitters. Future $\mathrm{THz}$ devices will be used for many different tasks. Besides detection of explosives, drugs and concealed weapon, one considers nowadays construction of systems for a short-range internet and television communication, as well as for a quick medical diagnosis of tissues during surgery operations. All these applications are based on optical arrangements including a $\mathrm{THz}$ source, a $\mathrm{THz}$ detector and an investigated object.

The interest in field-effect transistors (FETs) in connection with $\mathrm{THz}$ applications comes from the fact that they can be used both as emitters and detectors of this frequency. A scientific activity in this field started with theoretical models proposed by Dyakonov and Shur $[1,2]$ which implied a possibility of using FETs as detectors and emitters of $\mathrm{THz}$ radiation. The physical phenomenon lying behind this suggestion was two-dimensional (2D) electron plasma excitations and a non-linear character of the electron transport in the transistor channel.

For the last 10 years, many experimental, theoretical and Monte Carlo groups have been working on an analysis of plasma excitations in FETs, presenting new ideas and solutions. Looking at these devolopments one can expect that $\mathrm{THz}$ commercial devices based on FETs will soon be available, but one should also admit that there are many questions still unanswered which relate to the basic properties of a $2 \mathrm{D}$ plasma in spatially confined systems.

First experiments based on Dyakonov and Shur ideas exposed in Refs. [1, 2] were carried out at the beginning of this century and stimulated experimental and theoretical activities of many research groups around the world. The strongest driving force of the research was the hope to observe a resonant (i.e., spectrally narrow) and voltage-tunable response of FETs at room temperature that would be most suitable for applications. After a decade of research, one can formulate a few general state- ments which define the current status of FETs as THz devices. First, there is no doubt that a prevailing majority of FETs investigated so far allowed to detect and/or emit $\mathrm{THz}$ waves. Second, resonant features in the spectra are very weak and observed only at cryogenic temperatures. Third, there is a strong influence of the FET's metallization layout on the observed signal. Fourth, emerging applications are related to a room-temperature nonresonant detection.

The paper concentrates on one of the basic aspects of $\mathrm{THz}$ performance of FETs - the mechanism of photonplasmon coupling in a 2D plasma. The coupling in micrometer FETs is compared to that in large-area systems supplied with grid-gates which have served as standard structures to investigate plasma excitations for the last forty years. A role of a proper antenna for an effective $\mathrm{THz}$ performance of FETs is stressed.

\section{Photon-plasmon coupling in grid-gated large-area FETs}

First reports on plasma oscillations date back to the late 30ties [3] but it took some 30 years to start analyzing $2 \mathrm{D}$ systems. The first paper seems to be due to Stern [4] who analyzed the polarizability of a $2 \mathrm{D}$ conducting layer embedded in a uniform three-dimensional surrounding and calculated a dispersion relation $\omega(k)$ for plasma waves in this system ( $\omega$ is the angular frequency of oscillations and $k$ is the wave vector). Some years later, Chaplik [5] considered 2D plasma waves in connection with the Wigner crystallization in an electron layer on a surface of liquid helium.

The first experiment on a $2 \mathrm{D}$ electron plasma in a semiconductor system was carried out in 1972, and it contained many aspects that constitute the basis of such investigation up to now [6]. Before going into details, let us note that what we are thinking about is investigation of plasma excitations by optical experiments. This means that a basis for any observation is a photon-plasmon coupling which enables an excitation of the plasma by 
incident photons. This appears to be impossible in a translationally invariant system because the momentum of an incident photon propagating in such a medium is too small in comparison with that of a plasmon which is to be excited. A photon carries enough energy but its $\boldsymbol{k}$ vector is too small to fulfil the momentum conservation principle. In other words, on the $\omega-k$ plane a dispersion relation of a photon is situated to the left to that of a plasmon, while a photon-plasmon interaction requires a crossing of these two. Thus, one must think about an experimental configuration in which a photon would acquire an appropriate component of the in-plane momentum. A few experimental techniques were proposed and have been used until now [7] but the one that is most often adapted in experiments relies on preparation of a metallic grid with a periodicity $a$. This allows a diffraction of incident photons at angles that are defined by the in-plane $k$ vector component equal to $2 \pi n / a$, $n=0, \pm 1, \ldots$, corresponding to a diffraction pattern observed in the far-field. Putting it in more general terms, the idea is to prepare a periodic structure on the surface of the sample which was realized in a number of ways by different experimental groups $[8,9]$. In the presence of a periodic coupler, the wave vector of excited plasmons is equal to $2 \pi n / a$. A disadvantage of this method is that the plasmon wave vector is fixed for a given sample which essentially limits the ease of investigation of the $\omega(k)$ relation.

In the experiment of Ref. [6], a $\mathrm{Si}$ metal-oxidesemiconductor FET (MOSFET) with the surface area of a few $\mathrm{mm}^{2}$ was used with a metallic grating of $a=$ $3.52 \mu \mathrm{m}$ deposited on a gate. The transistor gate was used to change the concentration of the $2 \mathrm{D}$ electron gas $n_{\mathrm{s}}$ in the inversion layer thus changing the frequency of plasma oscillations. The Fourier spectroscopy transmission measurements, at a given gate polarization, revealed a structure corresponding to the absorption of the light by plasmons, which allowed to obtain an $\omega\left(n_{\mathrm{s}}\right)$ dependence at given $k$. Since then, these kinds of experiments on large-area FETs with periodic couplers have been carried out on a large variety of $2 \mathrm{D}$ systems allowing to establish a large part of our knowledge of a $2 \mathrm{D}$ plasma.

\section{Photon-plasmon coupling in micrometer FETs}

Let us note that in large-area grid-gated transistors exposed to the radiation incident photons are absorbed directly in the area occupied by a $2 \mathrm{D}$ plasma. This is not the case for small micrometer or nanometer FETs for which both the length and width of the channel are much smaller than the wavelength of the incoming radiation, and the channel is — at least partially - covered with a metallic gate. An absorption of photons directly in the channel is practically impossible. This problem was pointed out already in first papers by Dyakonov and Shur (Refs. [1, 2]) who proposed that the radiation couples to the channel through the contact pads. This means that incident photons are absorbed by metallic layers surrounding the channel and create a space- and time-dependent distribution of $\mathrm{THz}$ electric fields. These $\mathrm{THz}$ fields are a source of the force acting on a plasma confined in the channel. In their one-dimensional approach to this extremely complicated reality, Dyakonov and Shur proposed that the only relevant $\mathrm{THz}$ potential difference is that between the source and the gate, giving arguments based on a difference in capacitance between different contact pads of the transistor.

As a result of their analysis, Dyakonov and Shur proposed that the transistor channel is a resonator for plasma waves whose characteristic resonant frequencies can be tuned by the gate voltage. This result gave much hope for construction of voltage-tunable resonant $\mathrm{THz}$ devices, but this goal has not been achieved up to now: plasma resonances that are observed in FETs are weak and appear only at cryogenic temperatures. There are some physical reasons that can be responsible for this: too low electron mobility in the case of Si-MOSFETs, coupling of plasmons in gated and ungated parts in the case of high-electron mobility FETs or the presence of oblique modes (i.e., modes propagating at a certain angle to the source-drain direction).

We think that there can be also yet another reason, related to a very complex way in which a FET channel is excited. The importance of this factor was shown in a polarization experiment, where a response of a transistor to a linearly polarized $100 \mathrm{GHz}$ radiation was investigated as a function of the direction of the polarization plane [10]. A dipole-like response was observed and explained by numerical simulations of the electric field distribution around the FET. It was shown that the bonding wires and FETs metallic parts act as a voltage-tunable antenna, and the observed experimentally dipole dependence was due to that antenna, not to the channel itself. Numerical analysis shows also that at higher frequencies the incoming radiation generates a complicated pattern of the electric field in the substrate which then constitutes an essential part of the antenna.

One can give a simple analogy explaining why the way of excitation is important for observation of resonances. Let us imagine a single drop falling onto a flat water surface - a clear pattern of propagating waves will be created. Now, let us think about the rain - the created pattern of waves is chaotic and regular features of a single resonance are lost. We think that before stating final conclusions about possibilities to use plasma resonances in FETs one should solve the problem of the control of the channel excitations in real transistor structures. This can only be done by a careful design of an antenna concetrating the incoming radiation in a given, well defined region of the FET channel.

In spite of all these difficulties, there are some unquestionable facts concerning the detection with FETs. First, in spite of a lack of optimized antennas, FETs generally show a very strong, non-resonant signal that can be the basis of room temperature applications [11]. Second, ex- 
periments carried out at the magnetic fields clearly prove that the measured signal is related to a $2 \mathrm{D}$ plasma excitations [12], as is evidenced by observation of Shubnikov-de Haas oscillations and the cyclotron resonance transition in the detection signal.

\section{Experiments for applications}

$\mathrm{THz}$ detection and emission measurements were carried out on a large variety of FETs and in a variety of experimental conditions. From the material point of view, investigation concerned FETs fabricated on GaInAs/ AlInAs quantum wells, GaAs/AlGaAs and GaN/AlGaN heterostructures as well as on $\mathrm{Si} / \mathrm{SiO}_{2}$ inversion layers. Experiments were carried out at temperatures ranging from $4 \mathrm{~K}$ to $300 \mathrm{~K}$ with or without the magnetic field. A recent review paper [13] gives a fuller overview of results obtained. Here we would only like to put some attention to experiments carried out with Si-MOSFETs.

As it was mentioned above, the history of $2 \mathrm{D}$ plasma investigation in semiconductors started with measurements on Si-MOSFET [6], but the first detection experiments on nanometer Si-MOSFETs were carried out only at 2005 [14] and showed a possibility to use such FETs as room temperature detectors. The most important, however, seems to be showing up that the noise equivalent power of nanometer Si-MOSFETs can be as low as that of the best known $\mathrm{THz}$ detectors operating at room temperature [15]. Taking into account this result and all the extreme possibilities of the modern Si technology, one can seriously think about using Si-MOSFETs in commercial $\mathrm{THz}$ devices. In fact, first realizations of a few-pixel $\mathrm{THz}$ detectors - precursors of future $\mathrm{THz}$ video cameras has been shown operational recently [16]. It must be underlined that all emerging applications, based either on single FETs or arrays, use a nonresonant signal which typically grows strongly near the FET threshold voltage.

\section{Conclusions}

In the present paper we concentrated on a difference in the mechanism of coupling of $\mathrm{THz}$ photons with plasmons in large-area grid-gated structures and micrometer FETs. We underlined a crucial role of a complicated, antenna-like behavior of the metallization of contact pads in the case of FETs. We also suggested that the most promising future applications would be based on room-temperature nonresonant $\mathrm{THz}$ detection in Si-MOSFETs.

\section{Acknowledgments}

This work was partially supported by the Polish Ministry of Higher Education grants OR00015509 and N N515 071937.

\section{References}

[1] M. Dyakonov, M. Shur, Phys. Rev. Lett. 71, 2465 (1993).

[2] M. Dyakonov, M. Shur, IEEE Trans. Electron Dev. 43, 380 (1996).

[3] L. Tonks, I. Langmuir, Phys. Rev. 33, 195 (1929).

[4] F. Stern, Phys. Rev. Lett. 18, 546 (1967).

[5] V.A. Chaplik, Zh. Eksp. Teor. Fiz. 62, 746 (1972).

[6] S.J. Allen, D.C. Tsui, R.A. Logan, Phys. Rev. Lett. 38, 980 (1977).

[7] M.S. Kushwaha, Surf. Sci. Rep. 41, 1 (2001).

[8] U. Mackens, D. Heitmann, L. Prager, J. P. Kotthaus, W. Beinvogl, Phys. Rev. Lett. 53, 1485 (1984).

[9] R. Krahne, M. Hochgrafe, C. Heyn, D. Heitmann, M. Hauser, K. Eberl, Phys. Rev. B 62, 15345 (2000).

[10] M. Sakowicz, J. Łusakowski, K. Karpierz, M. Grynberg, W. Knap, W. Gwarek, J. Appl. Phys. 104, 024519 (2008).

[11] W. Knap, V. Kachorovskii, Y. Deng, S. Rumyantsev, J.-Q. Lü, R. Gaska, M. S. Shur, G. Simm, X. Hu, M. Asif Khan, C. A. Saylor, L. C. Brunel, J. Appl. Phys. 91, 9346 (2002).

[12] M. Sakowicz, J. Łusakowski, K. Karpierz, M. Grynberg, W. Knap, K. Kohler, G. Valusis, K. Gołaszewska, E. Kaminska, A. Piotrowska, Appl. Phys. Lett. 92, 203509 (2008).

[13] W. Knap, M. Dyakonov, D. Coquillat, F. Teppe, N. Dyakonova, J. Łusakowski, K. Karpierz, Sakowicz, G. Valusis, D. Seliuta, I. Kasalynas, A. El Fatimy, Y. M. Meziani, T. Otsuji, J. Infrared Milli Terahertz Wave 30, 1319 (2009).

[14] W. Knap, F. Teppe, Y. M. Meziani, N. Dyakonova, J. Łusakowski, F. Boeuf, T. Skotnicki, D. Maude, S. Rumyantsev, M.S. Shur, Appl. Phys. Lett. 85, 675 (2004).

[15] R. Tauk, F. Teppe, S. Boubanga, D. Coquillat, W. Knap, Y.M. Meziani, C. Gallon, F. Boeuf, T. Skotnicki, C. Fenouillet-Beranger, D.K. Maude, S. Rumyantsev, M.S. Shur, Appl. Phys. Lett. 89, 253511 (2006).

[16] E. Öjefors, U. Pfeiffer, A. Lisauskas, H. Roskos, IEEE J. Solid-State Circuits 44, 1 (2009). 